\begin{tabular}{|c|l|}
\hline Title & On the Stokes semigroup in some non-Helmholtz domains \\
\hline Author(s) & A be, Ken; GIGA, Y OSHIKAZU; Schade, K.; Suzuki, Takuya \\
\hline Citation & Hokkaido University Preprint Series in Mathematics, 1061, 2-10 \\
\hline Issue Date & 201410-20 \\
\hline DOI & 10.14943/84205 \\
\hline Doc URL & http://hdl.handle.net/2115/69865 \\
\hline Type & bulletin (article) \\
\hline File Information & pre1061.pdf \\
\hline
\end{tabular}

Instructions for use 


\title{
On the Stokes semigroup in some non-Helmholtz domains
}

\author{
Ken Abe, Yoshikazu Giga, Katharina Schade and Takuya Suzuki
}

\begin{abstract}
This paper shows that $L^{p}$-Helmholtz decomposition is not necessary to establish the analyticity of the Stokes semigroup in $C_{0, \sigma}$, the $L^{\infty}$-closure of the space of all compactly supported smooth solenoidal vector fields. In fact, in a sector-like domain for which the $L^{p}$-Helmholtz decomposition does not hold, the analyticity of the Stokes semigroup in $C_{0, \sigma}$ is proved.
\end{abstract}

Mathematics Subject Classification (2010). Primary 35J05; Secondary 35Q30, 76D07.

Keywords. Sector-like domain, Helmholtz decomposition, Neumann problem, weighted estimate.

\section{Introduction}

This paper is concerned with the Stokes semigroup; i.e., the solution operator $S(t): v_{0} \rightarrow v(, t)$ of the initial-boundary problem for the Stokes system

$$
v_{t}-\Delta v+\nabla q=0, \quad \operatorname{div} v=0 \quad \text { in } \quad \Omega \times(0, T)
$$

under zero Dirichlet boundary condition with initial condition $\left.v\right|_{t=0}=v_{0}$, where $\Omega$ is a domain in $\mathbf{R}^{n}$ with $n \geq 2$. It is well known that $S(t)$ forms an analytic semigroup in $L_{\sigma}^{p}(\Omega)(1<p<\infty)$ for various kind of domains $\Omega$ including smoothly bounded domains [13], [18], where $L_{\sigma}^{p}(\Omega)$ is the $L^{p}$-closure of $C_{c, \sigma}^{\infty}(\Omega)$, the space of all solenoidal vector fields with compact support in $\Omega$. In fact, the analyticity of $S(t)$ in $L_{\sigma}^{p}(\Omega)$ holds for any uniformly $C^{2}$-domain

This work was partly supported by the Japan Society for the Promotion of Science (JSPS) and the German Research Foundation through Japanese-German Graduate Externship and IRTG 1529. The work of Ken Abe is partly supported by JSPS as JSPS fellow. The work of Yoshikazu Giga is partly supported by JSPS through the grants Kiban S (26220702), Kiban A (23244015) and Houga (25610025). 
$\Omega$ provided that $L^{p}(\Omega)$ admits a topological direct sum decomposition, called the Helmholtz decomposition:

$$
L^{p}(\Omega)=L_{\sigma}^{p}(\Omega) \oplus G^{p}(\Omega), G^{p}(\Omega)=\left\{\nabla q \in L^{p}(\Omega) \mid q \in L_{l o c}^{p}(\Omega)\right\}
$$

This is recently proved in [12], where the maximal regularity in $L_{\sigma}^{p}(\Omega)$ is also established. The Helmholtz decomposition holds for any domain if $p=2$ and for various kind of domains like bounded or exterior domains with smooth boundary for $1<p<\infty$ [11]. However, for any $p>2$ there is an improper smooth sector-like domain such that the $L^{p}$-Helmholtz decomposition fails to satisfy [8], [15]. To be more precise, let $S_{\theta}$ denote $S_{\theta}=$ $\left\{x=\left(x_{1}, x_{2}\right)|| \arg x \mid<\theta / 2\right\}$, which is a sector in the plane $\mathbf{R}^{2}$ with opening angle $0<\theta<2 \pi$. We say that a planar domain $\Omega$ is a sector-like domain with opening angle $\theta$ if $\Omega \backslash D_{R}=S_{\theta} \backslash D_{R}$ for some $R>0$ (up to rotation and translation), where $D_{R}$ is an open disk of radius $R$ centered at the origin (figure 1).

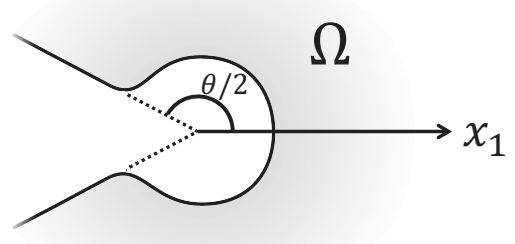

Figure 1

According to [15, Example 2, Fig. 5], the $L^{p}$-Helmholtz decomposition fails for a sector-like domain when $p>q_{\theta}^{\prime}$ or $p<q_{\theta}$ with $q_{\theta}=2 /(1+\pi / \theta)$ and $1 / q_{\theta}+1 / q_{\theta}^{\prime}=1$ even if it is smooth. (For $p \in\left(q_{\theta}, q_{\theta}^{\prime}\right)$ the $L^{p}$-Helmholtz decomposition holds [15].) Note that if the opening angle is larger than $\pi$, there always exists $p>2$ such that the $L^{p}$-Helmholtz decomposition fails.

The goal of this paper is to prove that the Stokes semigroup forms an analytic semigroup in $C_{0, \sigma}(\Omega)$ for a $C^{3}$ sector-like domain $\Omega$ for which the $L^{p}$-Helmholtz decomposition may fail. This shows that the existence of the $L^{p}$-Helmholtz decomposition may not be necessary for the analyticity of $S(t)$ in $C_{0, \sigma}(\Omega)$ although it is convenient to establish [3]. Note that the analyticity of $S(t)$ in $L_{\sigma}^{p}(\Omega)$ is not sufficient to guarantee its analyticity in $C_{0, \sigma}(\Omega)$ as shown in [19]. In fact, in [19] $S(t)$ is not analytic in $C_{0, \sigma}(\Omega)$ when $\Omega$ is an infinite layer domain in $\mathbf{R}^{n}(n \geq 3)$ while it is analytic in $L_{\sigma}^{p}(\Omega)(1<p<\infty)$; see e.g. [5], [6], [7]. (For a cylindrical domain (or an infinite cylinder) it is shown in [4] that $S(t)$ is analytic in $C_{0, \sigma}(\Omega)$ which is also analytic in $L_{\sigma}^{p}(\Omega)$ [10].) 
Theorem 1.1. Let $\Omega$ be a $C^{3}$ sector-like domain. Then $S(t)$ is a $C_{0}$-analytic semigroup in $C_{0, \sigma}(\Omega)$, the $L^{\infty}$-closure of $C_{c, \sigma}^{\infty}(\Omega)$. (Moreover, $t\left\|\nabla^{2} S(t) v_{0}\right\|_{\infty} /$ $\left\|v_{0}\right\|_{\infty}$ is bounded in $(0, T)$ where $\|\cdot\|_{\infty}$ denotes the supremum norm in $\Omega$.)

Interpolating $L^{\infty}$-result (Theorem 1.1) and $L^{2}$-result yields that the Stokes semigroup $S(t)$ is a $C_{0}$-analytic semigroup in a complex interpolated space $X_{p}=\left[L_{\sigma}^{2}(\Omega), C_{0, \sigma}(\Omega)\right]_{\theta}, 2 / p=1-\theta$. However, it is not clear that this space $X_{p}$ (continuously embedded in $L_{\sigma}^{p}(\Omega)$ ) agrees with $L_{\sigma}^{p}(\Omega)$.

By a recent result of [1] (see also [2], [3]) to show Theorem 1.1 it suffices to establish the next theorem which will be proved in the rest of this paper. Since we invoked the Hölder theory in [1], we need $C^{2, \gamma}$ regularity to apply results in [1]. This is a reason why we assume $C^{3}$ in Theorem 1.1 although it is not optimal at all. Note that it turns out that $C^{2}$ regularity is enough to prove the analyticity as in $[3$, Remark 1.5 (ii)].

Theorem 1.2. Let $\Omega$ be a $C^{2}$ sector-like domain. Then $\Omega$ is admissible in the sense of $[1$, Definition 2.3].

\section{Uniqueness for the Neumann problem}

We consider the uniqueness of the homogeneous Neumann problem

$$
\Delta u=0 \quad \text { in } \quad \Omega, \quad \frac{\partial u}{\partial n_{\Omega}}=0 \quad \text { on } \quad \partial \Omega,
$$

where $n_{\Omega}$ is the unit exterior normal vector field of $\partial \Omega$.

Lemma 2.1. Let $\Omega$ be a $C^{2}$ sector-like domain. Let $u \in C^{2}(\Omega) \cap C^{1}(\bar{\Omega})$ be $a$ solution of (2.1) satisfying

$$
\left\|d_{\Omega} \nabla u\right\|_{\infty}<\infty
$$

where $d_{\Omega}(x)=\inf _{y \in \partial \Omega}|x-y|$. Then $u$ is a constant function.

Lemma 2.2. Let $\Omega=S_{\theta}$. Let $u \in C^{2}(\Omega) \cap C^{1}(\bar{\Omega} \backslash\{0\})$ be a solution of (2.1) (except $x=0$ ) satisfying (2.2). Assume that for some $R>0$

$$
F(R):=\int_{\Gamma_{R} \cap \Omega} \frac{\partial u}{\partial r} d \mathcal{H}^{1}=0
$$

where $\Gamma_{R}=\partial D_{R}$ and $\partial / \partial r$ is the radial derivative. Then $u$ is a constant function.

Remark 2.3. The no flux condition (2.3) is necessary in Lemma 2.2. In fact $u=\log |x|$ solves $(2.1)$ with $(2.2)$ since $d_{\Omega}(x)=|x| \sin (\min ((\theta / 2-\varphi), \pi / 2))$ for $\varphi=\arg x>0$.

A key step for the proof of both Lemmas is to show boundedness of a solution. 
Lemma 2.4 (Boundedness). For $R>0$ let $u \in C^{2}\left(S_{\theta} \backslash D_{R}\right) \cap C^{1}\left(\bar{S}_{\theta} \backslash D_{R}\right)$ satisfy

$$
\Delta u=0 \quad \text { in } \quad S_{\theta} \backslash D_{R}, \quad \frac{\partial u}{\partial n_{\Omega}}=0 \quad \text { on } \quad\left(\partial S_{\theta}\right) \backslash D_{R} .
$$

Assume that $\left\|d_{S_{\theta}} \nabla u\right\|_{\infty}<\infty$ and $F\left(R_{1}\right)=0$ for some $R_{1}>R$. Then $u$ is bounded in $S_{\theta} \backslash D_{R+\delta}$ for any $\delta>0$.

Proof of Lemma 2.4. We may assume $R=1$ by dilation. We use polar coordinates $x_{1}=e^{s} \cos \varphi, x_{2}=e^{s} \sin \varphi$ so that $S_{\theta} \backslash D_{R}$ is transformed to a region $\{(s, \varphi)|s \geq 0,| \varphi \mid<\theta / 2\}$. The transformed dependent variable is denoted by $U$, i.e. $U(s, \varphi)=u\left(x_{1}, x_{2}\right)$. Then $U$ solves

$$
\Delta U=0 \text { in } \mathbf{R}_{+} \times\left(-\frac{\theta}{2}, \frac{\theta}{2}\right), \frac{\partial U}{\partial \varphi}=0 \text { on } \mathbf{R}_{+} \times\{ \pm \theta\}=\Gamma\left(\mathbf{R}_{+}=(0, \infty)\right)
$$

and satisfies

$$
|\nabla U| \leq C^{\prime} / d(\varphi), \quad d(\varphi)=\operatorname{dist}((s, \varphi), \Gamma)
$$

since $d_{S_{\theta}} \nabla u$ is bounded. Since $(2.4)$ holds, integration by parts shows that the flux $F\left(R^{*}\right)$ is independent of $R^{*}$. Thus $F\left(R^{*}\right)=0$ holds for all $R^{*}>1$, which yields $F\left(e^{s}\right)=d E(s) / d s=0$ for all $s>0$ with $E(s)=\int_{-\theta / 2}^{\theta / 2} U(s, \varphi) d \varphi$ since $r \partial / \partial r=\partial / \partial s$. Thus $E(s)$ is a constant $c$ independent of $s>0$. We may assume $c=0$ by subtracting $c$ from $U$. By integration of $\partial_{\varphi} U$ with respect to $\varphi$ variable $(2.6)$ implies that $U(s, \varphi)$ blows up at most logarithmically at $\pm \theta / 2$. By the uniform estimate (2.6) we observe that

$$
\sup _{S_{0}>0}\left\|U: L^{q}\left(\left(S_{0}, S_{0}+1\right) \times(-\theta / 2, \theta / 2)\right)\right\|<\infty
$$

for any $q>1$ (cf. [3]). By a standard elliptic regularity theory this implies that $U$ is bounded in $(\delta, \infty) \times(-\theta / 2, \theta / 2)$; see e.g. appendix of our companion paper [4].

Proof of Lemma 2.2. As in the proof of Lemma 2.4 we use the polar coordinates $(s, \varphi)$. We observe that $U$ satisfies $(2.5)$ in $\mathbf{R} \times(-\theta / 2, \theta / 2)$. By Lemma 2.4 we observe that $U$ is bounded for $s>1$. A similar argument implies that $U$ is also bounded for $s<-1$. Moreover, we may assume $E(s)=0$ for all $s>0$.

We shall prove that $U \equiv 0$ by the strong maximum principle $[17$, Ch. 2 , Sec. 3]. Assume that $U \not \equiv 0$. Then we may assume that $\sup U>0$ by considering $-U$ if necessary. This supremum is not attained in $\mathbf{R} \times[-\theta / 2, \theta / 2]$. Indeed, if it is attained in the interior, then the strong maximum principle implies that $U \equiv \sup U>0$ which contradicts the property $E(s)=0$. If the maximum is taken on the boundary, $U \equiv \sup U$ since otherwise the Hopf lemma [17, Ch. 2, Sec. 3, Thm. 7] implies $\partial U / \partial \varphi>0$ at that point which contradicts the Neumann condition. This again contradicts the property $E(s)=0$.

We may assume that there is a sequence $z_{m}=\left(s_{m}, \varphi_{m}\right)$ such that $U\left(z_{m}\right) \rightarrow \sup U$ and $\left|s_{m}\right| \rightarrow \infty$ as $m \rightarrow \infty$. We may assume that $s_{m} \rightarrow \infty$ since the case $s_{m} \rightarrow-\infty$ can be treated similarly. We may assume that 
$\varphi_{m} \rightarrow \varphi_{*}$ for some $\varphi_{*} \in[-\theta / 2, \theta / 2]$ by taking a subsequence. We shift $U$ and define $U_{m}(z):=U\left(s+s_{m}, \varphi\right)$ for $z=(s, \varphi)$ and observe that $\left\{U_{m}\right\}$ is a bounded sequence of solutions of (2.5) in $\mathbf{R} \times[-\theta / 2, \theta / 2]$. By Weyl's type lemma all derivatives are bounded so the Arzela-Ascoli theorem implies that $U_{m}$ converges to some solution $V$ of $(2.5)$ in $\mathbf{R} \times[-\theta / 2, \theta / 2]$ with its first derivatives locally uniformly in $\mathbf{R} \times[-\theta / 2, \theta / 2]$ by taking a subsequence. Then $V$ satisfies $\int_{-\theta / 2}^{\theta / 2} V(s, \varphi) d \varphi=0$ for all $s>0$ and $V\left(0, \varphi_{*}\right)=\max V=$ $\sup U>0$. As before the strong maximum principle and the Hopf lemma implies that $V \equiv \sup U>0$ which contradicts $\int_{-\theta / 2}^{\theta / 2} V(s, \varphi) d \varphi=0$. We thus conclude that $U \equiv 0$.

Proof of Lemma 2.1. By the assumption $\Omega \backslash D_{R_{0}}=S_{\theta} \backslash D_{R_{0}}$ for some $R_{0}>0$. By (2.1) we observe that $F(R)=0$ for all $R>R_{0}$. By Lemma 2.4, we see that $u$ is bounded in $\bar{\Omega}$. As in the proof of Lemma 2.4 we use the polar coordinates $(s, \varphi)$. We observe that $U$ satisfies $(2.5)$ in $\left(\log R_{0}, \infty\right) \times(-\theta / 2, \theta / 2)$. By the no flux condition $F(R)=0$ we may assume that $E(s)=0$ for $s \in\left(\log R_{0}, \infty\right)$ by adding a constant.

As in the proof of Lemma 2.2 one is able to prove that $u \equiv 0$ by a minor modification.

\section{Weighted $L^{\infty}$ estimates for the Neumann problem}

We are interested in a priori estimates for a weak solution of the Neumann problem. Let $\Omega$ be a $C^{2}$-domain in $\mathbf{R}^{n}(n \geq 1)$. Let $g \in L_{l o c}^{1}(\partial \Omega)$ be a tangential vector field, i.e. $g \cdot n_{\Omega}=0$ on $\partial \Omega$. We say that $u \in L_{l o c}^{1}(\bar{\Omega})$ is a weak solution of

$$
\Delta u=0 \quad \text { in } \quad \Omega, \quad \frac{\partial u}{\partial n_{\Omega}}=\operatorname{div}_{\partial \Omega} g
$$

if $u$ satisfies

$$
\int_{\Omega} u \Delta \varphi d x=\int_{\partial \Omega} \nabla_{\partial \Omega} \varphi \cdot g d \mathcal{H}^{1}
$$

for all $\varphi \in C_{c}^{2}(\bar{\Omega})$ with $\frac{\partial \varphi}{\partial n_{\Omega}}=0$ on $\partial \Omega$, where $\operatorname{div}_{\partial \Omega}=\nabla_{\partial \Omega}$. denotes the surface divergence [4], where $\nabla_{\partial \Omega}=P_{\partial \Omega} \nabla$ and $P_{\partial \Omega}$ is the tangential projection, i.e., $P_{\partial \Omega}=I-n_{\Omega} \otimes n_{\Omega}$. This definition is essentially given in [1] and is the same as in [2]. Note that the tangential gradient $\nabla_{\partial \Omega}$ can be replaced by $\nabla$ since $g$ is tangential. The main feature of this definition is that $u$ can be unbounded near $\partial \Omega$. Such a notion of weak solutions are elaborated by $[16]$ to include the case that the Neumann data contains Dirac measure.

Lemma 3.1. Let $\Omega$ be a $C^{2}$ sector-like domain in $\mathbf{R}^{2}$. Then there exists a constant $C$ independent of $R \geq 1$ such that the estimate

$$
\left\|d_{\Omega}(x) \nabla u\right\|_{\infty} \leq C\|g\|_{\infty}
$$

holds for all weak solution $u \in L_{\text {loc }}^{1}\left(\bar{\Omega}_{R}\right)$ of (3.1) in $\Omega_{R}=D_{2 R} \cap \Omega$ with $g \in L^{\infty}\left(\partial \Omega_{R}\right)$ satisfying $g \cdot n_{\Omega}=0$ on $\left(\partial \Omega_{R}\right) \backslash \Omega$ and $g=0$ on $\partial \Omega_{R} \cap \Omega$ provided that $\left\|d_{\Omega} \nabla u\right\|_{\infty}<\infty$. 
Proof of Lemma 3.1. Although the proof is similar to [4, Lemma 2.5], we give it for completeness. As in [1], [2], we argue by contradiction. Suppose that (3.3) were false. Then there would exist $\left\{u_{m}, g_{m}, R_{m}\right\}_{m=1}^{\infty}$ satisfying

$$
1=\left\|d_{\Omega} \nabla u_{m}\right\|_{L^{\infty}\left(\Omega_{R_{m}}\right)}>m\left\|g_{m}\right\|_{L^{\infty}\left(\partial \Omega \cap D_{2 R_{m}}\right)}
$$

such that $u_{m} \in L_{\text {loc }}^{1}\left(\bar{\Omega}_{R_{m}}\right)$ is a weak solution of (3.1) in $\Omega_{R}$ with $g_{m} \in$ $L^{\infty}\left(\partial \Omega_{R_{m}}\right)$ satisfying $g_{m} \cdot n_{\Omega}=0$ on $\partial \Omega \cap D_{2 R}$ and $g_{m}=0$ on $\partial D_{2 R} \cap \Omega$. We take $x_{m} \in \bar{\Omega}_{R_{m}}$ such that

$$
\left|d_{\Omega}\left(x_{m}\right) \nabla u_{m}\left(x_{m}\right)\right|>1 / 2 .
$$

We may assume that $u_{m}\left(x_{m}\right)=0$ by adding a constant.

There are two cases depending on the behavior of $\left\{x_{m}\right\}_{m=1}^{\infty}$.

Case 1 . There exists a subsequence still denoted by $\left\{x_{m}\right\}$ which converges to $\hat{x} \in \bar{\Omega}$ as $m \rightarrow \infty$.

Case 2. The sequence $\left\{x_{m}\right\}$ tends to infinity, i.e. $\left|x_{m}\right| \rightarrow \infty$.

We discuss Case 1 which is divided into two cases, (a) $\hat{x} \in \Omega$ and (b) $\hat{x} \in \partial \Omega$. We may assume that $R_{m} \rightarrow R \in[1, \infty]$ by taking a subsequence. In the case (a) by (3.4) it is easy to prove that $\left\{u_{m}\right\}$ converges to a weak solution of (3.1) in $\Omega^{\infty}=\Omega_{R}$ if $R<\infty$ and $=\Omega$ if $R=\infty$ with $g=0$ by taking a subsequence. Moreover, the convergence is locally uniform with its derivatives in $\Omega^{\infty}$ so that $u(\hat{x})=0$, since $\left\{u_{m}\right\}$ is harmonic and bounded in $L_{l o c}^{q}(\bar{\Omega})$ for all $q \geq 1$ by (3.4) and $u_{m}\left(x_{m}\right)=0$. If $R$ is finite, then the elliptic regularity [4, Appendix A] implies that $u \in C^{\infty}\left(\Omega^{\infty}\right) \cap C^{1}\left(\overline{\Omega^{\infty}}\right)$. Although there are two corner points in $\Omega^{\infty} \cap\{|x|=2 R\}$, one can show that $u$ is smooth up to these points by reflection in $s$ of $(s, \varphi)$-variable in the proof of Lemma 2.4 since the Neumann data at $|x|=2 R$ is zero. The uniqueness (up to constant) of the Neumann problem in this domain is easy to prove as in Lemma 2.1 by the strong maximum principle. We thus conclude that $u \equiv 0$. However, by (3.5) we have $\left|d_{\Omega}(\hat{x}) \nabla u(\hat{x})\right| \geq 1 / 2$, which yields a contradiction. If $R=\infty$, then we apply Lemma 2.1 to conclude $u \equiv 0$, which yields a contradiction. The case (b) can be treated as in [1] by rescaling $u_{m}$ as

$$
v_{m}(x)=u_{m}\left(x_{m}+d_{m} x\right)
$$

with $d_{m}=d_{\Omega}\left(x_{m}\right)$. (We only need $C^{2}$-regularity of $\Omega$ as in [4] in this step.) We apply the uniqueness result in a half space [1, Lemma 2.9] to get a contradiction. If $R$ is finite, then there might be a chance that the rescaled limit space (obtained as a limit of $\Omega_{m}=\left\{x \mid x_{m}+d_{m} x \in \Omega_{R_{m}}\right\}$ ) is not a half space but a quadrant type space $\left\{x_{2}>0, x_{1}<R\right\}$. In this case we extend a solution by even reflection outside $x_{1}=R$ and reduce the problem in the half space.

We next study Case 2. We rescale $u_{m}$ as

$$
w_{m}(x)=u_{m}\left(\left|x_{m}\right| x\right)
$$

and set $y_{m}=x_{m} /\left|x_{m}\right|, H_{m}=R_{m} /\left|x_{m}\right|$. We may assume that $H_{m} \rightarrow H \in$ $[1, \infty]$. Then $\left\{w_{m}\right\}$ converges to a weak solution $w$ of $(3.1)$ in $\Omega^{\infty}=S_{\theta} \cap D_{2 H}$ with $g=0$ by taking a subsequence. We have to divide the case depending 
on $y_{m} \rightarrow \hat{y} \in \Omega^{\infty}$ and $\hat{y} \in \partial \Omega^{\infty}$. The second case can be handled by rescaling $w_{m}$ of (3.7) by (3.6) and reduce the problem to the uniqueness in the half space. The first case is more involved. The limit $w$ of $\left\{w_{m}\right\}$ must satisfy

$$
|\nabla w(x)| \leq C / d_{S_{\theta}}(x), \quad x \in \Omega^{\infty}=S_{\theta} \cap D_{2 H} .
$$

One would like to apply the uniqueness in $\Omega^{\infty}$ with (3.8). In the case $H<\infty$, the uniqueness result like Lemma 2.2 can be proved since the no flux condition (2.3) is automatically fulfilled. The case $H=\infty$ needs to prove the no flux condition (2.3). We introduce a cut-off function $\eta_{k}(k=1,2, \ldots)$ defined by $\eta_{k}(x)=\eta(k(|x|-1 / 2)+1 / 2)$ where $\eta \in C^{2}[0, \infty]$ satisfies $\eta(s)=0$ for $0 \leq s \leq 1 / 2$ and $\eta(s)=1$ for $s \geq 1$ with $0 \leq \eta \leq 1$ and $\eta^{\prime} \geq 0$. Since $w_{m}$ is a weak solution of $(3.1)$ in $\Omega^{m}=\Omega_{R_{m}} /\left|x_{m}\right|$ with $g=\tilde{g}_{m}, \tilde{g}_{m}(x)=g_{m}\left(\left|x_{m}\right| x\right)$, we observe that

$$
\int_{\Omega_{m} \cap D_{1 / 2}^{c}} w_{m} \Delta \eta_{k} d x=\int_{\partial \Omega_{m} \cap D_{2 H_{m}}} \nabla_{\partial \Omega} \eta_{k} \cdot \tilde{g}_{m} d \mathcal{H}^{1} .
$$

Since $\left\|\tilde{g}_{m}\right\|_{\infty}=\left\|g_{m}\right\|_{\infty} \leq 1 / m$ by (3.4), sending $m \rightarrow \infty$ implies

$$
\int_{S_{\theta} \cap D_{1 / 2}^{c}} w \Delta \eta_{k} d x=0
$$

Integrating by parts yields

$$
k \int_{1 / 2}^{1 / 2+1 / k}\left(\int_{\Gamma_{r} \cap S_{\theta}} \frac{\partial w}{\partial r} d \mathcal{H}^{1}\right) \eta^{\prime}(k(r-1 / 2)+1 / 2) r d r=0 .
$$

Sending $k \rightarrow \infty$ yields the no flux condition

$$
\int_{\Gamma_{1 / 2} \cap S_{\theta}} \frac{\partial w}{\partial r} d \mathcal{H}^{1}=0 .
$$

By (3.9) one is able to apply Lemma 2.2 with $w(\hat{y})=0$ to conclude that $w \equiv 0$ while $(3.5)$ implies $\left|d_{S_{\theta}}(\hat{y}) \nabla w(\hat{y})\right|>1 / 2$ which is a contradiction.

Theorem 3.2. Let $\Omega$ be a $C^{2}$ sector-like domain in $\mathbf{R}^{2}$. Then there exists a constant $C$ such that (3.3) holds for all weak solution $u \in L_{\text {loc }}^{1}(\bar{\Omega})$ of (3.1) with $\nabla u \in L^{2}(\Omega)$ and $g \in L^{\infty}(\partial \Omega)$ with $g \cdot n_{\Omega}=0$ on $\partial \Omega$.

As in the proof [2, Proposition 2.6] that strictly admissibility implies the admissibility, Theorem 3.2 implies Theorem 1.2.

Remark 3.3. (i) The estimate (3.3) is very similar to saying that $\Omega$ is strictly admissible in the sense of [2]. However, there is an important difference. In Theorem 3.2, we restrict $u$ such that $\nabla u$ is globally square integrable. So Theorem 3.2 does not assert that $\Omega$ is strictly admissible.

(ii) To show admissibility in [1] we invoked $C^{3}$-regularity of a domain. This is because we have used $C^{3}$-regularity to prove the uniqueness of the Neumann problem as well as the flattening procedure as in the proof of handling case (b) below. However, in the present paper uniqueness results in Section 2 require only $C^{2}$-regularity. If one examines carefully as in [4], the flattening procedure requires only $C^{2}$-regularity so do Lemma 3.1 and Theorem 3.2. 
Proof of Theorem 3.2. Let $R_{0}>0$ such that $\Omega \backslash D_{R_{0}}=S_{\theta} \backslash D_{R_{0}}$. For $R>R_{0}$ let $w_{R}$ be a solution of the Neumann problem

$$
\Delta w_{R}=0 \text { in } \Omega_{R}, \quad \frac{\partial w_{R}}{\partial n_{\Omega}}=0 \text { on }\left(\partial \Omega_{R}\right) \backslash \Omega, \quad \frac{\partial w}{\partial r}=\frac{\partial u}{\partial r} \text { on } \partial \Omega_{R} \cap \Omega .
$$

Since $\nabla u \in L^{2}(\Omega)$ so that $\frac{\partial u}{\partial r} \in L^{2}\left(\partial \Omega_{R} \cap \Omega\right)$ for almost all $R>R_{0}$ by the Lax-Milgram theorem, this problem admits a solution $w_{R}$ (unique up to constant) with $\nabla w_{R} \in L^{2}\left(\Omega_{R}\right)$ for almost all $R>R_{0}$. We shall consider such $R$ in the sequel.

We set $u_{R}=u-w_{R}$ and observe that

$$
\left\|d_{\Omega} \nabla u_{R}\right\|_{L^{\infty}\left(\Omega_{R}\right)} \leq C\|g\|_{\infty}
$$

by Lemma 3.1 since $\nabla u_{R} \in L^{2}\left(\Omega_{R}\right)$ implies $d_{\Omega}\left|\nabla u_{R}\right| \in L^{\infty}\left(\Omega_{R}\right)$ for a harmonic $u_{R}$ by two dimensionality; see [1, Remark 2.4 (ii)].

If we prove that $\nabla w_{R} \rightarrow 0$ in $L^{2}(\Omega)$, then the desired estimate follows from (3.10) by the lower semicontinuity of $\left\|d_{\Omega} \nabla u\right\|_{\infty}$ with respect to $L^{2}$ convergence $\nabla u_{R} \rightarrow \nabla u$.

It remains to prove that $\nabla w_{R} \rightarrow 0$ in $L^{2}(\Omega)$ as $R \rightarrow \infty$ by taking a subsequence. It is convenient to introduce $(s, \varphi)$ coordinates as in the proof of Lemma 2.4. We observe that

$\int_{S_{\theta} \backslash D_{R_{0}}}|\nabla f|^{2} d x_{1} d x_{2}=\int_{W}\left|\nabla_{s, \varphi} \tilde{f}\right|^{2} d s d \varphi, \quad W=\left(\log R_{0}, \infty\right) \times(-\theta / 2, \theta / 2)$, where $\tilde{f}(s, \varphi)=f\left(e^{s} \cos \varphi, e^{s} \sin \varphi\right)$. By definition we have

$$
\int_{\Omega_{R}}\left|\nabla w_{R}\right|^{2} d x_{1} d x_{2}=\int_{|x|=2 R} \frac{\partial w_{R}}{\partial r} w_{R} d \mathcal{H}^{1}=\int_{|x|=2 R} \frac{\partial u}{\partial r} w_{R} d \mathcal{H}^{1} .
$$

We use $(s, \varphi)$ coordinates to get

$$
\int_{\Omega_{R}}\left|\nabla w_{R}\right|^{2} d x_{1} d x_{2}=\left.\int_{-\theta}^{\theta} e^{-s} \frac{\partial \tilde{u}}{\partial s} \tilde{w}_{R} e^{s}\right|_{s=\log 2 R} d \varphi=\left.\int_{-\theta}^{\theta} \frac{\partial \tilde{u}}{\partial s} \tilde{w}_{R}\right|_{s=\log 2 R} d \varphi .
$$

Since $w_{R}$ satisfies the no flux condition, we may assume that $\int_{-\theta / 2}^{\theta / 2} \tilde{w}_{R} d \varphi=0$ at $s=\log 2 R$. By the Poincaré inequality and the trace theorem [9] we have

$$
\int_{-\theta / 2}^{\theta / 2}\left|\tilde{w}_{R}\right|^{2} d \varphi \leq C \int_{W_{R}}\left|\nabla_{s, \varphi} \tilde{w}_{R}\right|^{2} d \varphi d s, \quad W_{R}=\left(\log R_{0}, \log 2 R\right) \times(-\theta / 2, \theta / 2)
$$

with $C$ independent of $R$. By the Hölder inequality (3.11) now yields

$$
\int_{\Omega_{R}}\left|\nabla w_{R}\right|^{2} d x_{1} d x_{2} \leq\left(\left.\int_{-\theta / 2}^{\theta / 2}\left|\frac{\partial \tilde{u}}{\partial s}\right|^{2} d \varphi\right|_{s=\log 2 R}\right)^{1 / 2}\left(C \int_{\Omega_{R}}\left|\nabla w_{R}\right|^{2} d x_{1} d x_{2}\right)^{1 / 2} .
$$

This implies

$$
\int_{\Omega_{R}}\left|\nabla w_{R}\right|^{2} d x_{1} d x_{2} \leq\left. C \int_{-\theta / 2}^{\theta / 2}\left|\frac{\partial \tilde{u}}{\partial s}\right|^{2} d \varphi\right|_{s=\log 2 R}
$$


Since $\nabla u \in L^{2}(\Omega)$ so that $\nabla_{s, \varphi} \tilde{u} \in L^{2}(W)$, the right-hand side of (3.12) tends to zero as $R \rightarrow \infty$ by taking a suitable subsequence. Thus (3.12) implies that $\nabla w_{R} \rightarrow 0$ in $L^{2}(\Omega)$ (by interpreting $\nabla w_{R}=0$ outside $\Omega_{R}$ ) for a subsequence $R \rightarrow \infty$.

\section{References}

[1] K. Abe and Y. Giga, Analyticity of the Stokes semigroup in spaces of bounded functions. Acta Math. 211 (2013), 1-46.

[2] K. Abe and Y. Giga, The $L^{\infty}$-Stokes semigroup in exterior domains. J. Evol. Equ. 14 (2014), no. 1, 1-28.

[3] K. Abe, Y. Giga and M. Hieber, Stokes resolvent estimates in space of bounded functions. Annales scientifiques de l'ENS, to appear.

[4] K. Abe, Y. Giga, K. Schade and T. Suzuki, On the Stokes resolvent estimates for domains with finitely many outlets. in preparation.

[5] T. Abe and Y. Shibata, On a resolvent estimate of the Stokes equation on an infinite layer. J. Math. Soc. Japan 55 (2003), 469-497.

[6] T. Abe and Y. Shibata, On a resolvent estimate of the Stokes equation on an infinite layer. II. J. Math. Soc. Japan 5 (2003), 245-274.

[7] H. Abels and M. Wiegner, Resolvent estimates for the Stokes operator on an infinite layer. Diff. Int. Eqs. 18 (2005), 1081-1110.

[8] M. E. Bogovskiǐ, Decomposition of $L_{p}\left(\Omega, R^{n}\right)$ into the direct sum of subspaces of solenoidal and potential vector fields. Dokl. Akad. Nauk. SSSR 286 (1986), 781786 (Russian); English translation in Soviet Math. Dokl. 33 (1986), 161-165.

[9] L. C. Evans, Partial Differential Equations. Second edition, American Mathematical Society, Providence, Rhode Island, 2010.

[10] R. Farwig and M.-H. Ri, Resolvent estimates and maximal regularity in weighted $L^{q}$-spaces of the Stokes operator in an infinite cylinder. J. Math. Fluid Mech. 10 (2008), 352-387.

[11] G. P. Galdi, An Introduction to the Mathematical Theory of the Navier-Stokes Equations. Vol. I. Linearized Steady Problems. Springer Tracts in Natural Philosophy 38, Springer, New York, 1994.

[12] M. Geissert, H. Heck, M. Hieber and O. Sawada, Weak Neumann implies Stokes. J. Reine Angew. Math. 669 (2012), 75-100.

[13] Y. Giga, Analyticity of the semigroup generated by the Stokes operator in $L_{r}$ spaces. Math. Z. 178 (1981), 297-329.

[14] Y. Giga, Surface Evolution Equations: A level set approach. Monographs in Mathematics 99 Birkhäuser, Basel-Boston-Berlin, 2006, xii+264pp.

[15] V. N. Maslennikova and M. E. Bogovskii, Elliptic boundary value problems in unbounded domains with noncompact and non smooth boundaries. Rend. Sem. Mat. Fis. Milano 56 (1986), 125-138.

[16] J. Merker and J.-M. Rakotoson, Very weak solutions of Poisson's equation with singular data under Neumann boundary conditions. Calc. Var. and PDEs, to appear. 
[17] M. H. Protter and H. F. Weinberger, Maximum principles in differential equations. Prentice-Hall, Englewood Cliffs, New Jersey, 1967, reprented by SpringerVerleg, 1984, New York.

[18] V. A. Solonnikov, Estimates for solutions of nonstationary Navier-Stokes equations. J. Soviet Math. 8 (1977), 467-529.

[19] L. von Below, The Stokes and Navier-Stokes equations in layer domains with and without a free surface. Thesis, Technische Universität Darmstadt (2014).

Ken Abe

Nagoya University

Furocho, Chikusa-ku, Nagoya

Aichi 464-8602,

Japan

e-mail: abe.ken@math.nagoya-u.ac.jp

Yoshikazu Giga

University of Tokyo

3-8-1 Komaba, Meguro-ku

Tokyo 153-8914,

Japan

e-mail: labgiga@ms.u-tokyo.ac.jp

Katharina Schade

Technische Universität Darmstadt

Fachbereich Mathematik, Schlossgartenstr. 7

D-64298 Darmstadt,

Germany

e-mail: schade@mathematik.tu-darmstadt.de

Takuya Suzuki

University of Tokyo

3-8-1 Komaba, Meguro-ku

Tokyo 153-8914,

Japan

e-mail: tsuzuki@ms.u-tokyo.ac.jp 